\title{
Soft due window assignment and scheduling of unit-time jobs on parallel machines
}

\author{
Adam Janiak • Wladyslaw Janiak • \\ Mikhail Y. Kovalyov • Frank Werner
}

Received: 22 October 2011 / Revised: 2 April 2012 / Published online: 13 May 2012

(C) The Author(s) 2012. This article is published with open access at Springerlink.com

\begin{abstract}
We study problems of scheduling $n$ unit-time jobs on $m$ identical parallel machines, in which a common due window has to be assigned to all jobs. If a job is completed within the due window, then no scheduling cost incurs. Otherwise, a job-dependent earliness or tardiness cost incurs. The job completion times, the due window location and the size are integer valued decision variables. The objective is to find a job schedule as well as the location and the size of the due window such that a weighted sum or maximum of costs associated with job earliness, job tardiness and due window location and size is minimized. We establish properties of optimal solutions of these min-sum and min-max problems and reduce them to min-sum (traditional) or min-max (bottleneck) assignment problems solvable in $O\left(n^{5} / \mathrm{m}^{2}\right)$ and $O\left(n^{4.5} \log ^{0.5} n / m^{2}\right)$ time, respectively. More efficient solution procedures are given
\end{abstract}

\footnotetext{
A. Janiak $(\varangle)$

Institute of Computer Engineering, Control and Robotics, Wrocław University of Technology, Janiszewskiego 11/17, 50-372 Wrocław, Poland

e-mail: adam.janiak@pwr.wroc.pl

W. Janiak

Faculty of Computer Science and Management, Wrocław University of Technology, Łukasiewicza 5, 50-371 Wrocław, Poland e-mail: wladyslaw.janiak@pwr.wroc.pl

M. Y. Kovalyov

United Institute of Informatics Problems, National Academy of Sciences of Belarus, Surganova 6, 220012 Minsk, Belarus

e-mail: kovalyov_my@newman.bas-net.by

F. Werner

Faculty of Mathematics, Otto-von-Guericke-University Magdeburg, PSF 4120, 39016 Magdeburg, Germany

e-mail: frank.werner@mathematik.uni-magdeburg.de
} 
for the case in which the due window size cost does not exceed the due window start time cost, the single machine case, the case of proportional earliness and tardiness costs and the case of equal earliness and tardiness costs.

Keywords Scheduling - Parallel machines - Earliness-tardiness · Due window · Unit-time jobs

\section{MSC classification $\quad 68 \mathrm{M} 20$}

\section{Introduction}

We study problems that combine parallel machine scheduling of unit-time jobs with due window assignment. A due window is a time interval associated with a job. No cost is incurred if a job is completed within its due window. Otherwise, an earliness or tardiness cost is incurred. The problems can be formulated as follows.

There are $n$ independent non-preemptive jobs to be scheduled for processing on $m$ parallel machines. Each machine can handle at most one job at a time and each job can be completely processed on any machine. All jobs have unit processing requirements and a common due window $[e, d], e \leq d$, is assigned to all jobs, where $e$ and $d$ are decision variables to be determined. A schedule determines an allocation of the jobs to the machines and the job starting and completion times. Given a schedule, denote the completion time of job $j$ by $C_{j}, j=1, \ldots, n$. Earliness and tardiness of job $j$ is determined as $E_{j}=\max \left\{0, e-C_{j}\right\}$ and $T_{j}=\max \left\{0, C_{j}-d\right\}$, respectively. Job $j$ with $E_{j}>0, T_{j}>0$ and $E_{j}=T_{j}=0$ is called early, tardy, and on-time, respectively. The objective is to find an optimal schedule and the optimal values of the decision variables so as to minimize a sum $F_{\Sigma}=\sum_{j=1}^{n}\left\{\alpha_{j} E_{j}+\beta_{j} T_{j}\right\}+\gamma e+\delta(d-e)$ or a maximum $F_{\max }=\max _{1 \leq j \leq n}\left\{\alpha_{j} E_{j}+\beta_{j} T_{j}+\gamma e+\delta(d-e)\right\}$. Here $\alpha_{j}, \beta_{j}$, $j=1, \ldots, n, \gamma$ and $\delta$ are given non-negative numbers, which determine the costs of job earliness, job tardiness, due window start time and due window size, respectively. We assume that all the job completion times $C_{j}$ and the decision variables $e$ and $d$ take non-negative integer values. Further, we assume without loss of generality that $e \geq 1$. It is easy to see that equality $e=0$ is satisfied in an optimal solution only if $\gamma \geq \delta$, in which case an optimal solution for the problem with the constraint $e \geq 1$ can be converted into an optimal solution of the original problem by decreasing the values of $e, d$ and all $C_{j}$ by one.

Because there are two types of objective functions, we consider two problems, which we denote as P-sum and P-max. Descriptors "max" and "sum" reflect the type of the objective function.

The concept of the due window generalizes the concept of the due date. It first appeared in the vehicle routing literature, see Schrage (1981), Bodin et al. (1983), Kritikos and Ioannou (2010), Li et al. (2010), Hashimoto et al. (2010) and Liberatore et al. (2011). The due window concept was introduced to the scheduling research area by Cheng (1988). In both areas, hard and soft due windows are distinguished, see Solomon and Desrosiers (1988) and Biskup and Feldmann (2005). In the hard due window case, job completion before or after its due window is prohibited. In contrast, 
the soft due window concept assumes that earlier or later job completion is possible at an additional cost. In this paper, we consider the soft due window case.

Scheduling problems with due window assignment can be used for mathematical modeling of product delivery dates negotiation between the manufacturer and the customers in a make-to-order manufacturing system, see Wang et al. (1998) and Wang et al. (1999). Biskup and Feldmann (2005) pointed out that small buffer stocks exist in most companies - even in those which have adopted the just-in-time principle- to adjust to a slightly earlier or later delivery of ordered goods. Lee (1991) observed due window scheduling problems in metal cutting companies and electronic companies. If the due window is tight, the problems appear in semiconductor manufacturing, see Anger et al. (1986). Koulamas (1997) discussed applications of a due window model in scheduling chemical reactions and satellite photography, Yeung et al. (2004) in scheduling assembly lines, Janiak et al. (2007) in construction industry, Wen et al. (2010) in crane scheduling.

The specificity of the problems P-sum and P-max is the unit-time jobs and the common due window. It is typical for manufacturing similar products on a demand of the same customer. Consider a manufacturer which produces paving tiles on parallel lines to be used in the same project of a construction company. The corresponding demand consists of a number of pallets each of which can be considered as a job. The construction company and the manufacturer negotiate the due window for the delivery of all the pallets. An early delivery is often preferable to begin the project earlier. A smaller due window size is also preferable to minimize the time dependent cost of the resources required for the receipt of the pallets. These preferences lead to the due window costs $\gamma$ and $\delta$. The two parties also agree about the relative importance of the pallets with respect to their completion before or after the due window because of the relative urgency of the corresponding construction works. The latter leads to the earliness and tardiness costs. Then the manufacturer needs to solve the problem P-sum if minimizing the total cost is the objective, or the problem P-max if minimizing the largest individual cost is the objective.

A recent bibliography of the results on scheduling problems with various models of due date and due window assignment was provided by Gordon et al. (2002), Chu et al. (2002), Yen and Wan (1999) and Kanet and Sridharan (2000). Most of the results on scheduling with hard or soft job due windows were obtained for a single machine processing environment. To the best of our knowledge, a combination of parallel machine scheduling with due window assignment was studied only by Kramer and Lee (1994), Mosheiov (2001), Mosheiov and Oron (2004), Janiak et al. (2007) and Mosheiov and Sarig (2010). Kramer and Lee (1994) studied a problem with min-sum objective, in which the due window is given and job processing times are arbitrary. They proved NP-hardness of this problem, presented a dynamic programming algorithm for the two-machine case and a heuristic for the general case. Mosheiov (2001) and Mosheiov and Oron (2004) considered problems with cost coefficients $\alpha_{j}=\alpha$ and $\beta_{j}=\beta$ for all jobs. Mosheiov (2001) proposed a heuristic algorithm and a lower bound for a min-max problem with arbitrary job processing times, while Mosheiov and Oron (2004) suggested a constant time solution algorithm for a min-sum problem with unit-time jobs, which is a special case of problem P-sum. Janiak et al. (2007) considered common due window models with no due window start time cost $(\delta=0)$, 
$\alpha_{j}=\alpha, \beta_{j}=\beta$ for all jobs, and arbitrary job processing times. For min-max and min-sum problems, they established properties of optimal solutions and proved that problems with unit cost coefficients are polynomially equivalent to the classical parallel machine scheduling problem to minimize the makespan. Mosheiov and Sarig (2010) suggested a linear programming formulation for a problem with arbitrary job processing times and given job sequences on the machines. Some of the results related to our studies appeared earlier in conference proceedings, see Janiak and Winczaszek (2003).

Our study is also related to the studies of common due date scheduling problems. Here the most recent contributions by Kellerer and Strusevich (2006); Karakostas et al. (2009); Kacem (2010) and Tuong et al. (2010) can be mentioned.

In the following sections, we establish properties of optimal solutions for the considered problems. Section 3 considers the case of arbitrary earliness/tardiness costs and $m$ machines. Solution procedures for the single machine case and the case of proportional earliness/tardiness costs are described in Sect. 4. The paper concludes with a summary of the results, a discussion of possible generalizations and suggestions for further research in Sect. 5.

\section{Properties of optimal solutions}

Given a solution to problem P-max or P-sum, let $C_{\max }$ denote the completion time of the latest job. We prove the following properties.

Theorem 1 There exists an optimal solution for problems $P$-max and $P$-sum, which possesses the following properties

(i) there is no machine idle time between the jobs on the same machine;

(ii) at least one machine starts at time zero;

(iii) $d-e \leq\left\lceil\frac{n}{m}\right\rceil-1$;

(iv) no job is completed outside the due window if there is an unoccupied time slot in the interval $[e-1, d]$ on a machine;

(v) one of the following two cases holds:

(a) $[e, d]=\left[1, C_{\max }\right]$, each machine starts at time 0 and completes at a time $t \in\left\{C_{\max }-1, C_{\max }\right\}$, where $C_{\max }=\left\lceil\frac{n}{m}\right\rceil$, or

(b) $[e, d] \neq\left[1, C_{\max }\right]$, each machine starts at a time $t_{1} \in\{0,1\}$, completes at a time $t_{2} \in\left\{C_{\max }-1, C_{\max }\right\}$, where $C_{\max } \in\left\{\left\lceil\frac{n}{m}\right\rceil,\left\lceil\frac{n}{m}\right\rceil+1\right\}$ and time interval $[e-1, d]$ is fully occupied on each machine. If additionally $\delta \leq \gamma$, then $e=1$, each machine starts at time zero, $C_{\max }=\left\lceil\frac{n}{m}\right\rceil$ and $d \leq C_{\max }$.

Proof Consider an optimal schedule and assume that property (i) is not satisfied: there exist two jobs $i$ and $j$ scheduled in this order on the same machine and there is an idle time between them. At least one of the following two modifications of the schedule does not increase the objective function value: (1) shifting the job sequence on the left of job $i$ including job $i$ to the right, or (2) shifting the job sequence on the right of job $j$ including job $j$ to the left. Therefore, a schedule with no machine idle time between jobs $i$ and $j$ is optimal. Repetition of this shifting argument a finite number of times proves property (i). 


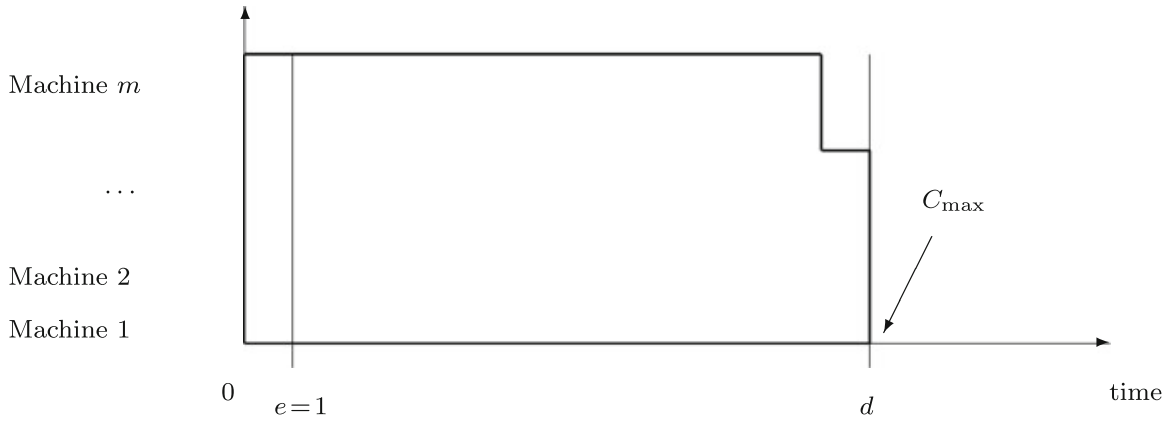

Fig. 1 Schedule of the first type: $e=1, d=\left\lceil\frac{n}{m}\right\rceil=C_{\max }$

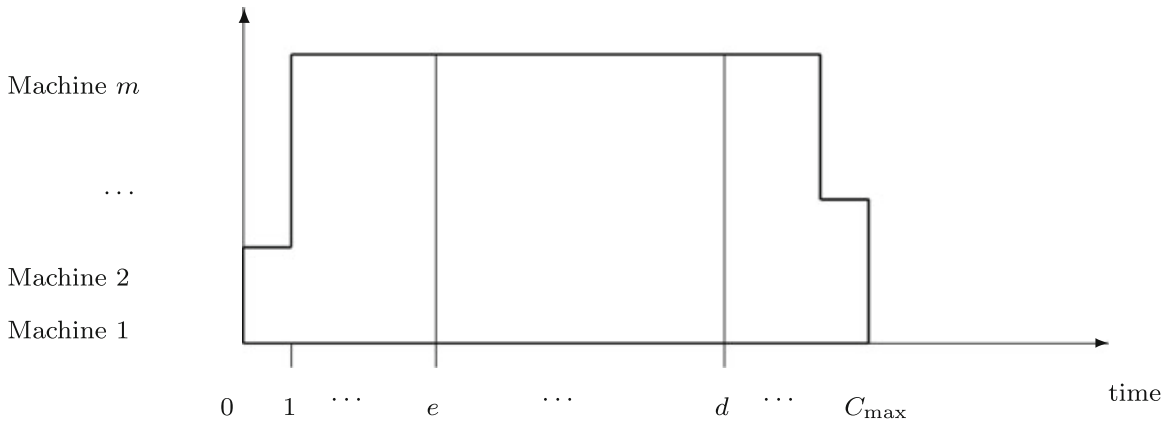

Fig. 2 Schedule of the second type: $C_{\max } \in\left\{\left\lceil\frac{n}{m}\right\rceil,\left\lceil\frac{n}{m}\right\rceil+1\right\}$, interval $[e-1, d]$ is fully occupied

Property (ii) is proved by an observation that shifting all the jobs and the due window to the left does not change earliness/tardiness and due window size costs and decreases (if $\gamma>0$ ) or does not change (if $\gamma=0$ ) the due window start time cost. If the due window is in the left most position $(e=1)$ and no machine starts at time zero, the jobs can be shifted to the left so that property (ii) is satisfied.

To prove property (iii), assume that there exists an optimal solution with a due window satisfying $d-e \geq\left\lceil\frac{n}{m}\right\rceil$. The cost of this solution is at least $\delta\left\lceil\frac{n}{m}\right\rceil+\gamma$ for any of the problems P-sum and P-max. Consider another solution, in which $e=1, d=\left\lceil\frac{n}{m}\right\rceil$ and all the jobs are completed by $d$. In the corresponding schedule, $x$ machines process $\left\lceil\frac{n}{m}\right\rceil-1$ jobs each and $m-x$ machines process $\left\lceil\frac{n}{m}\right\rceil$ jobs each starting at time 0 , where $x$ is determined from the equality $x\left(\left\lceil\frac{n}{m}\right\rceil-1\right)+(m-x)\left\lceil\frac{n}{m}\right\rceil=n$, i.e., $x=m\left\lceil\frac{n}{m}\right\rceil-n$. The cost of this solution is equal to $\delta\left(\left\lceil\frac{n}{m}\right\rceil-1\right)+\gamma$ for any of the two problems, which contradicts the optimality of the solution with $d-e \geq\left\lceil\frac{n}{m}\right\rceil$, unless $\delta=0$. The case $\delta=0$ is trivial.

If there is a job completed outside the due window and there is an unoccupied time slot in the interval $[e-1, d]$ on a machine, then moving this job to be processed within this time slot on this machine does not increase the objective function value, which proves property (iv).

The succeeding proof is supported by Figs. 1 and 2, where examples of optimal solutions of two possible types corresponding to cases (a) and (b) in property (v) are given. 
Observe that there exist only two types of optimal solutions satisfying properties (i)-(iv). For a first type solution, all the jobs are completed within the due window, and for a second type solution, there are jobs completed outside the due window. Any first type solution can be modified without affecting optimality so that case a) of property (v) takes place, see Fig. 1 .

Consider a second type solution. Note that the time interval $[e-1, d]$ is fully occupied on each machine due to property (iv) and that the time slot $[0,1]$ is occupied on some machine due to property (ii). If there is an unoccupied time slot $[t-1, t]$, $2 \leq t \leq e-1$, on a machine, then a job processed in the time slot $[0,1]$ can be moved to the time slot $[t-1, t]$ without affecting optimality. Therefore, we can assume that either all the machines start at the same time or their start times differ by one. Since shifting the whole schedule and the due window to the left does not change optimality, we can assume that each machine starts at a time $t_{1} \in\{0,1\}$. The time interval $[e-1, d]$ remains fully occupied on each machine. Consider such an optimal schedule. If there is an unoccupied time slot $[t, t+1], d \leq t \leq C_{\max }-2$, on some machine, then a job processed in the time slot $\left[C_{\max }-1, C_{\max }\right]$ can be moved to the time slot $[t, t+1]$ without affecting optimality. Therefore, we can assume that each machine completes at a time $t_{2} \in\left\{C_{\max }-1, C_{\max }\right\}$, where $C_{\max } \in\left\{\left\lceil\frac{n}{m}\right\rceil,\left\lceil\frac{n}{m}\right\rceil+1\right\}$. The time interval $[e-1, d]$ remains fully occupied on each machine. The constructed optimal solution satisfies case b) of property (v), see Fig. 2 .

Finally, if $\delta \leq \gamma$ and $e \geq 2$, then re-setting $e=1$ does not increase objective function value. Optimal solution with $e=1$ can be further transformed into an optimal solution, in which each machine starts at time zero, $C_{\max }=\left\lceil\frac{n}{m}\right\rceil$ and $d \leq C_{\max }$.

From now on, we limit ourselves to solutions satisfying properties (i)-(v).

\section{Arbitrary earliness/tardiness costs and $m$ machines}

Due to property (v), there can be optimal solutions to problems P-sum and P-max of two types corresponding to cases (a) and (b) of this property, respectively. Examples are given in Figs. 1 and 2.

The cost of an optimal solution in Fig. 1 is equal to $\gamma+\delta\left(\left\lceil\frac{n}{m}\right\rceil-1\right)$ for any of the problems P-sum and P-max. In the rest of the paper, we consider an optimal solution in Fig. 2.

\subsection{The case $\delta \leq \gamma$}

In the considered case, each machine starts at time zero, $e=1, C_{\max }=\left\lceil\frac{n}{m}\right\rceil$ and $d \leq C_{\max }$ in an optimal solution for any of the problems $\mathrm{P}$-sum and $\mathrm{P}$-max. In this solution, there is no early job, tardy jobs have minimal values $\beta_{j}$ and are assigned to time intervals $\left[\left\lceil\frac{n}{m}\right\rceil-1,\left\lceil\frac{n}{m}\right\rceil\right],\left[\left\lceil\frac{n}{m}\right\rceil-2,\left\lceil\frac{n}{m}\right\rceil-1\right], \ldots,[d, d+1]$ in the non-decreasing order of $\beta_{j}$. The cost of this solution, denoted as $F_{x}^{(\delta \leq \gamma)}(d)$, where $x \in\{\Sigma$, $\max \}$, depends solely on $d$. Re-number the jobs such that $\beta_{1} \leq \cdots \leq \beta_{n}$.

For problem P-sum, define $\beta_{k}^{*}$ as the sum of $\beta_{j}$ values of the jobs assigned as described above to time interval $[k-1, k]: \beta_{k}^{*}=\sum_{j=1}^{m+n-m\left\lceil\frac{n}{m}\right\rceil} \beta_{j}$ for $k=\left\lceil\frac{n}{m}\right\rceil$, and 
$\beta_{k}^{*}=\sum_{j=(k-1) m+1}^{k m} \beta_{j}$ for $1 \leq k \leq\left\lceil\frac{n}{m}\right\rceil-1$. In this case, $F_{\Sigma}^{(\delta \leq \gamma)}(d)=\gamma+\delta(d-$ $1)+S(d)$, where $S(d)$ is the contribution of the tardy jobs, which depends solely on $d$ and can be calculated as $S(d)=\sum_{k=d+1}^{\left\lceil\frac{n}{m}\right\rceil}(k-d) \beta_{k}^{*}$. We have $F_{\Sigma}^{(\delta \leq \gamma)}\left(\left\lceil\frac{n}{m}\right\rceil\right)=$ $\gamma+\delta\left(\left\lceil\frac{n}{m}\right\rceil-1\right)$ and $F_{\Sigma}^{(\delta \leq \gamma)}(d)-F_{\Sigma}^{(\delta \leq \gamma)}(d-1)=\delta-B(d)$, where $B(d)$ is the total weight of the jobs assigned to time intervals $[d-1, d], \ldots,\left[\left\lceil\frac{n}{m}\right\rceil-1,\left\lceil\frac{n}{m}\right\rceil\right], B(d)=$ $\beta_{d}^{*}+\beta_{d+1}^{*}+\cdots+\beta_{\left\lceil\frac{n}{m}\right\rceil}^{*}$, for $d=2,3, \ldots,\left\lceil\frac{n}{m}\right\rceil$. Since $\beta_{1}^{*} \geq \cdots \geq \beta_{\left\lceil\frac{n}{m}\right\rceil}^{*}, B(d)$ is convexly decreasing in $d$ and $F_{\Sigma}^{(\delta \leq \gamma)}(d)$ is discretely convex in $d$. Therefore, it can be minimized in $O\left(\frac{n}{m} \log \frac{n}{m}\right)$ time by a tripartite search like golden ratio or Fibonacci search, see Torn and Zilinskas (1989), in the range of feasible $d$ values.

For problem P-max, re-define $\beta_{k}^{*}$ as the largest $\beta_{j}$ value of the jobs assigned to time interval $[k-1, k]: \beta_{k}^{*}=\max _{1 \leq j \leq m+n-m\left\lceil\frac{n}{m}\right\rceil}\left\{\beta_{j}\right\}$ for $k=\left\lceil\frac{n}{m}\right\rceil$, and $\beta_{k}^{*}=$ $\max _{(k-1) m+1 \leq j \leq k m}\left\{\beta_{j}\right\}$ for $1 \leq k \leq\left\lceil\frac{n}{m}\right\rceil-1$. Then $F_{\max }^{(\delta \leq \gamma)}(d)=\gamma+\delta(d-1)+$ $M(d)$, where $M(d)$ is the contribution of the tardy jobs to the objective function of problem P-max. This contribution depends on $d$ alone, and it can be calculated as $M(d)=\max _{d+1 \leq k \leq\left\lceil\frac{n}{m}\right\rceil}\left\{(k-d) \beta_{k}^{*}\right\}$. In this case, $F_{\max }^{(\delta \leq \gamma)}\left(\left\lceil\frac{n}{m}\right\rceil\right)=F_{\text {sum }}^{(\delta \leq \gamma)}\left(\left\lceil\frac{n}{m}\right\rceil\right)=$ $\gamma+\delta\left(\left\lceil\frac{n}{m}\right\rceil-1\right)$ and $F_{\max }^{(\delta \leq \gamma)}(d)-F_{\max }^{(\delta \leq \gamma)}(d-1)=\delta-(M(d-1)-M(d))$ for $d=2,3, \ldots,\left\lceil\frac{n}{m}\right\rceil$. Contrary to problem P-sum, function $M(d-1)-M(d)$ is not convexly decreasing and $F_{\max }^{(\delta \leq \gamma)}(d)$ is not discretely convex in $d$ in general. Since all the values $M(d)$ can be found in $O\left(\frac{n^{2}}{m^{2}}\right)$ time, $F_{\max }^{(\delta \leq \gamma)}(d)$ can be minimized in $O\left(\frac{n^{2}}{m^{2}}\right)$ time.

Thus, in the case $\delta \leq \gamma$ problem P-sum can be solved in $O(n \log n)$ time, and problem P-max in $O\left(\max \left\{n \log n, \frac{n^{2}}{m^{2}}\right\}\right)$ time.

\subsection{The case $\delta \leq \gamma$}

Let values $e$ and $d$ be given. Denote the corresponding problems P-sum and P-max as $\mathrm{P}(e, d)$-sum and $\mathrm{P}(e, d)$-max. Introduce $0-1$ variables $x_{j l t}$ and coefficients $a_{j l t}, j=$ $1, \ldots, n, l=1, \ldots, m$ and $t=1,2, \ldots, C_{\max }$ such that

$$
\begin{aligned}
& x_{j l t}= \begin{cases}1, & \text { if job } j \text { is scheduled in the time slot }[t-1, t] \text { on machine } l, \\
0, & \text { otherwise, }\end{cases} \\
& a_{j l t}= \begin{cases}(e-t) \alpha_{j}, & \text { if } 1 \leq t \leq e-1, \\
0, & \text { if } e \leq t \leq d, \\
(t-d) \beta_{j}, & \text { if } d+1 \leq t \leq C_{\max } .\end{cases}
\end{aligned}
$$

Problem $\mathrm{P}(e, d)$-sum reduces to the following ( min-sum) assignment problem:

$$
\text { Minimize } \sum_{j=1}^{n} \sum_{l=1}^{m} \sum_{t=1}^{C_{\max }} a_{j l t} x_{j l t},
$$


subject to

$$
\begin{aligned}
& \sum_{l=1}^{m} \sum_{t=1}^{C_{\max }} x_{j l t}=1 \text { for all } j, \\
& \sum_{j=1}^{n} x_{j l t} \leq 1 \quad \text { for all }(l, t), \\
& x_{j l t} \in\{0,1\} \quad \text { for all }(j, l, t) .
\end{aligned}
$$

Problem $\mathrm{P}(e, d)$-max reduces to the following bottleneck (min-max) assignment problem:

$$
\text { Minimize } \max _{1 \leq j \leq n} \max _{1 \leq l \leq m} \max _{1 \leq t \leq C_{\max }} a_{j l t} x_{j l t} \text {, subject to (1)-(3). }
$$

The min-sum assignment problem can be solved in $O\left(n^{3}\right)$ time and the minmax assignment problem can be solved in $O\left(n^{2.5} \log ^{0.5} n\right)$ time, see Burkard and Cela (1999). Since we need to enumerate $O\left(n^{2} / m^{2}\right)$ appropriate pairs $(e, d)$, problems P-sum and P-max can be solved in $O\left(n^{5} / m^{2}\right)$ and $O\left(n^{4.5} \log ^{0.5} n / m^{2}\right)$ time, respectively.

\section{Proportional earliness/tardiness costs and single machine}

We consider two special cases: the single machine case and the identical parallel machine case with proportional earliness/tardiness costs $\beta_{j}=r \alpha_{j}, j=1, \ldots, n$, where $r>0$ is a given number. The single machine case is of practical interest if the corresponding production environment consists of a single facility or if the original problem can be decomposed into several single machine problems without much loss in the quality of the combined decision. Since the single machine problems are often easier solvable, the decomposition approach is widely used in practice. The same motivation is for considering proportional earliness and tardiness costs - the corresponding problem is easier and, if the solution time is important, the original problem can be relaxed to fit into this easier formulation.

\subsection{Single machine case}

Let us consider the single machine case, which we denote as P1-sum and P1-max depending on the type of the objective function.

Consider an optimal solution in Fig. 2. Observe that in order to find it, we do not need to enumerate values $e$ and $d$ because each job assigned as early contributes one unit to the due window start time $e$ and each job assigned as on-time, except for the job completing at $e$, contributes one unit to the due window size $(d-e)$. The on-time job completing at $e$ does not contribute to $(d-e)$ but it contributes to $e$. Clearly, the specificity of this on-time job is immaterial. 
Introduce sets $E, I$ and $T$ of early, on-time and tardy jobs, respectively. Introduce variables $x_{j S t}$ and coefficients $a_{j S t}, j=1, \ldots, n, S \in\{E, I, T\}, t=1, \ldots, n$, such that

$$
\begin{aligned}
& x_{j E t}= \begin{cases}1, & \text { if } j \in E \text { and it is scheduled in position } t<e-1, \\
0, & \text { otherwise, }\end{cases} \\
& x_{j I t}= \begin{cases}1, & \text { if } j \in I, \\
0, & \text { otherwise, }\end{cases} \\
& x_{j T t}= \begin{cases}1, & \text { if } j \in T \text { and it is scheduled in position } t>d, \\
0, & \text { otherwise, }\end{cases} \\
& a_{j S t}= \begin{cases}t \alpha_{j}+\gamma, & \text { if } S=E, \\
\delta, & \text { if } S=I, \\
t \beta_{j}, & \text { if } S=T .\end{cases}
\end{aligned}
$$

Problem P1-sum reduces to the following (min-sum) assignment problem:

$$
\operatorname{Minimize} \sum_{j=1}^{n} \sum_{S \in\{E, I, T\}} \sum_{t=1}^{n} a_{j S t} x_{j S t}+\gamma-\delta \text {, }
$$

subject to

$$
\begin{array}{cl}
\sum_{S \in\{E, I, T\}} \sum_{t=1}^{n} x_{j S t}=1 & \text { for all } j, \\
\sum_{j=1}^{n} x_{j S t} \leq 1 & \text { for all }(S, t), \\
x_{j S t} \in\{0,1\} & \text { for all }(j, S, t) .
\end{array}
$$

The term $\gamma-\delta$ in the objective function is introduced to adjust the contribution of the specific on-time job completing at $e$ : we deduct $\delta$ to obtain its zero total contribution to $(d-e)$ and add $\gamma$ to count its extra contribution to $e$.

Problem P1-max reduces to the following bottleneck (min-max) assignment problem.

$$
\text { Minimize } \max _{1 \leq j \leq n} \max _{S \in\{E, I, T\}} \max _{1 \leq t \leq n} a_{j} x_{j} x_{j t}+\gamma-\delta \text {, subject to (4)-(6). }
$$

The latter min-sum and min-max assignment problems can be solved in $O\left(n^{3}\right)$ and $O\left(n^{2.5} \log ^{0.5} n\right)$ time, respectively, which are the time complexities of solving the corresponding problems P1-sum and P1-max. 
Li et al. (2008) suggested an $O\left(n^{4} \log n\right)$ algorithm for a modification of problem $\mathrm{P} 1$-max, in which $\delta$ is sufficiently large number such that $d=e$ in any optimal solution and $d$ can be fractional.

\subsection{Proportional earliness/tardiness costs}

In the considered case, there are $m$ identical parallel machines and $\beta_{j}=r \alpha_{j}$ for $j=1, \ldots, n$. Let $e$ and $d$ be given. For an optimal solution in Fig. 2, we say that a job is in positions $(E, t),(I, t)$ and $(T, t)$ if it is early, on-time and tardy, respectively, and is scheduled in $t$-th time slot on one of the machines.

Consider positional weights

$$
w_{S t}:= \begin{cases}t, & \text { if } S=E, \\ 0, & \text { if } S=I, \\ t r, & \text { if } S=T .\end{cases}
$$

Since each position can be occupied by $m$ jobs, consider $m$ copies of each positional weight and renumber them to use a single index, which we also call a position: $w_{1}, w_{2}, \ldots$

Problems $\mathrm{P}(e, d)$-sum and $\mathrm{P}(e, d)$-max with fixed values $e$ and $d$ reduce to finding $n$ optimal positions $i_{1}^{*}, \ldots, i_{n}^{*}$ and an assignment of jobs to these positions such that $\sum_{j=1}^{n} \alpha_{j} w_{i_{j}^{*}}$ and $\max _{1 \leq j \leq n} \alpha_{j} w_{i_{j}^{*}}$ is minimized, respectively. Observe that the optimal positions are those corresponding to the $n$ smallest positional weights, and they can be selected in $O(n)$ time by merging three ordered sequences of positional weights: $(0, \ldots, 0),(1, \ldots, 1,2, \ldots, 2, \ldots)$ and $(r, \ldots, r, 2 r, \ldots, 2 r, \ldots)$. Here the first sequence contains $y=m(d-e)$ smallest weights $w_{I t}=0$, the second sequence contains $\min \{m(e-1), n-y\}$ smallest weights $w_{E t}=k, l=1, \ldots, m, k=$ $1, \ldots, e-1$, and the third sequence contains $\min \left\{m\left(C_{\max }-d\right), n-y\right\}$ smallest weights $w_{T t}=t r, l=1, \ldots, m, t=1, \ldots, C_{\max }-d$. Note that $y$ is a multiple of $m$ because for an optimal solution in Fig. 2 the time interval $[e-1, d]$ is fully occupied on each machine. The total number of positions to be considered is equal to $y+\min \{m(e-1), n-y\}+\min \left\{m\left(C_{\max }-d\right), n-y\right\} \leq 2 n-y \leq 2 n$. After the $n$ optimal positions (with smallest weights) have been found, $\sum_{j=1}^{n} \alpha_{j} w_{i_{j}^{*}}$ and $\max _{1 \leq j \leq n} \alpha_{j} w_{i_{j}^{*}}$ are minimized by matching the $n$ smallest positional weights with the $n$ largest values $\alpha_{j}$. The time requirement of this matching procedure is determined by sorting the values $\alpha_{j}$ in a non-decreasing order, which can be done in $O(n \log n)$ time. Therefore, problems $\mathrm{P}(e, d)$-sum and $\mathrm{P}(e, d)$-max with proportional earliness/tardiness costs can be solved in $O(n)$ time, provided that a non-decreasing sequence of the $\alpha_{j}$ values is given. We deduce that by enumerating appropriate pairs $(e, d)$, problems P-sum and P-max with the proportional earliness/tardiness costs can be solved in $O\left(n^{3} / m^{2}\right)$ time. Note that the non-decreasing sequence of $\alpha_{j}$ values needs to be constructed only once.

The indicated time requirement can be improved if the minimum value of the earliness/tardiness part of the objective function $\left(F_{\Sigma}\right.$ or $\left.F_{\max }\right)$ convexly decreases as 
Table 1 Computational complexity of problems P-sum and P-max

\begin{tabular}{lll}
\hline Problem notation & Conditions & Complexity \\
\hline P-sum & & $O\left(n^{5} / m^{2}\right)$ \\
P-sum & $\delta \leq \gamma$ & $O(n \log n)$ \\
P-sum & $\beta_{j}=r \alpha_{j}$ & $O\left(n^{3} / m^{2}\right)$ \\
P-sum & $\beta_{j}=\alpha_{j}$ & $O\left(n^{2} \log \frac{n}{m}\right)$ \\
P1-sum & & $O\left(n^{3}\right)$ \\
P-max & & $O\left(n^{4.5} \log 0.5 n / m^{2}\right)$ \\
P-max & $\delta \leq \gamma$ & $O\left(\max \left\{n \log n, \frac{n^{2}}{m^{2}}\right\}\right)$ \\
P-max & $\beta_{j}=r \alpha_{j}$ & $O\left(n^{3} / m^{2}\right)$ \\
P-max & $\beta_{j}=\alpha_{j}$ & $O\left(n^{2} \log \frac{n}{m}\right)$ \\
P1-max & & $O\left(n^{2.5} \log 0.5 n\right)$ \\
\hline
\end{tabular}

$d$ increases. This is the case if $r=1$, i.e., $\alpha_{j}=\beta_{j}, j=1, \ldots, n$, see "Appendix" for a proof. In this case, the minimum value of the objective function will be convex in $d$, and its global minimum can be found by a tripartite search in $O\left(n^{2} \log \frac{n}{m}\right)$ time. For $r \neq 1$, convexity in $d$ of the minimum value of the earliness/tardiness part of $F_{\Sigma}$ or $F_{\max }$ is not known.

\section{Conclusions}

We have studied problems P-sum and P-max of scheduling $n$ unit-time jobs on $m$ identical parallel machines, in which a common due window has to be assigned to all jobs and there are job-dependent earliness and tardiness costs and due window location and size costs. These problems can be used for mathematical modeling of product delivery dates negotiation between the manufacturer and the customers in a make-to-order manufacturing system. The results of this paper are summarized in Table 1.

Our results can be generalized to the case of job-and machine-dependent earliness and tardiness costs. While some of the properties established in Theorem 1 do not hold for this case, a reduction to the assignment problems and polynomial time solution procedures are still possible because it can be easily proved that $d \leq n$ and, given $e$ and $d$, the number of appropriate positions for job processing is $O(n)$ on each machine.

Further research can be undertaken to investigate unit-time scheduling problems for soft due window models different from the common due window model considered in this paper. Slack due window and processing-plus-weight due window models are more general and can be of interest. In these models, due windows are job-dependent such that job $j$ is assigned a due window $\left[e_{j}, d_{j}\right]$ with $e_{j}=p_{j}+e, d_{j}=p_{j}+d, 0 \leq e \leq d$, for the slack due window model, and with $e_{j}=k p_{j}+e, d_{j}=k p_{j}+d, 0 \leq e \leq$ $d, k \geq 0$, for the processing-plus-weight due window model. Parameters $e, d$ and $k$ are the decision variables. 
Open Access This article is distributed under the terms of the Creative Commons Attribution License which permits any use, distribution, and reproduction in any medium, provided the original author(s) and the source are credited.

\section{Appendix A. The case of equal earliness and tardiness costs}

Consider the case $\alpha_{j}=\beta_{j}, j=1, \ldots, n$. Let $\alpha_{1} \leq \cdots \leq \alpha_{n}$. Denote the minimum value of the earliness/tardiness part of $F_{\Sigma}$ and $F_{\max }$ as a function of $d$ by $E T_{\Sigma}^{*}(d)$ and $E T_{\text {max }}^{*}(d)$, respectively.

Lemma 1 If $\alpha_{j}=\beta_{j}, j=1, \ldots, n$, then $E T_{\Sigma}^{*}(d)$ and $E T_{\max }^{*}(d)$ are convex.

Proof Consider the feasible values $d, d+1$ and $d+2$. We will show that $E T_{\Sigma}^{*}(d)+$ $E T_{\Sigma}^{*}(d+2) \geq 2 E T_{\Sigma}^{*}(d+1)$ and $E T_{\max }^{*}(d)+E T_{\max }^{*}(d+2) \geq 2 E T_{\max }^{*}(d+1)$, which is sufficient for the proof. The discussion of job positional weights in Sect. 4 implies that

$$
\begin{gathered}
E T_{\Sigma}^{*}(d)=1 \sum_{i=k_{d}-m+1}^{k_{d}} \alpha_{i}+2 \sum_{i=k_{d}-2 m+1}^{k_{d}-m} \alpha_{i}+\cdots+\left(q_{d}-1\right) \sum_{i=m_{d}+1}^{m_{d}+m} \alpha_{i}+q_{d} \sum_{i=1}^{m_{d}} \alpha_{i}, \\
E T_{\max }^{*}(d)=\max \left\{1 \max _{k_{d}-m+1 \leq i \leq k_{d}} \alpha_{i}, 2 \max _{k_{d}-2 m+1 \leq i \leq k_{d}-m} \alpha_{i}, \ldots,\left(q_{d}-1\right)\right. \\
\left.\max _{m_{d}+1 \leq i \leq m_{d}+m} \alpha_{i}, q_{d} \max _{1 \leq i \leq m_{d}} \alpha_{i}\right\},
\end{gathered}
$$

where the positive integer numbers $k_{d}, m_{d}$ and $q_{d}, k_{d} \leq n, m_{d} \leq m, q_{d} \leq\left\lfloor\frac{n}{m}\right\rfloor$, depend on $d$. Furthermore,

$$
\begin{aligned}
E T_{\Sigma}^{*}(d+1)= & 1 \sum_{i=k_{d}-2 m+1}^{k_{d}-m} \alpha_{i}+2 \sum_{i=k_{d}-3 m+1}^{k_{d}-2 m} \alpha_{i}+\cdots+\left(q_{d}-2\right) \\
& \times \sum_{i=m_{d}+1}^{m_{d}+m} \alpha_{i}+\left(q_{d}-1\right) \sum_{i=1}^{m_{d}} \alpha_{i}, \\
E T_{\max }^{*}(d+1)= & \max \left\{1 \max _{k_{d}-2 m+1 \leq i \leq k_{d}-m} \alpha_{i}, 2 \max _{k_{d}-3 m+1 \leq i \leq k_{d}-2 m} \alpha_{i}, \ldots,\right. \\
E T_{\Sigma}^{*}(d+2)= & \left.1 \sum_{i=k_{d}-3 m+1}^{\left(q_{d}-2\right)} \max _{m_{d}+1 \leq i \leq m_{d}+m} \alpha_{i},\left(q_{d}-1\right) \max _{1 \leq i \leq m_{d}} \alpha_{i}\right\}, \\
& \times \sum_{i=k_{d}-3 m+1}^{m_{d}+m} \alpha_{i} \alpha_{i}+\left(q_{d}-2\right) \sum_{i=1}^{m_{d}} \alpha_{i},
\end{aligned}
$$




$$
\begin{gathered}
E T_{\max }^{*}(d+2)=\max \left\{{ }^{1} \max _{k_{d}-3 m+1 \leq i \leq k_{d}-2 m} \alpha_{i}, 2 \max _{k_{d}-4 m+1 \leq i \leq k_{d}-3 m} \alpha_{i}, \ldots,\right. \\
\left.\left(q_{d}-3\right) \max _{m_{d}+1 \leq i \leq m_{d}+m} \alpha_{i},\left(q_{d}-2\right) \max _{1 \leq i \leq m_{d}} \alpha_{i}\right\} .
\end{gathered}
$$

Let the cost coefficient $\alpha_{i}, 1 \leq i \leq n$, be multiplied by a number $i_{d}$ in the formulas for $E T_{\Sigma}^{*}(d)$ and $E T_{\max }^{*}(d)$. If $\alpha_{i}$ is not present in the corresponding formula, then $i_{d}=0$. It can easily be seen that the following three cases are the only possible: 1 ) $\left.i_{d}=i_{d+1}=i_{d+2}=0,2\right) i_{d}=1, i_{d+1}=i_{d+2}=0$, and 3) $i_{d} \geq 2, i_{d+1}=i_{d}-1$, $i_{d+2}=i_{d}-2$. In either case, $\left(i_{d}+i_{d+2}\right) \geq 2 i_{d+1}$, which implies that $E T_{\Sigma}^{*}(d)+$ $E T_{\Sigma}^{*}(d+2) \geq 2 E T_{\Sigma}^{*}(d+1)$ and $E T_{\max }^{*}(d)+E T_{\max }^{*}(d+2) \geq 2 E T_{\max }^{*}(d+1)$.

\section{References}

Anger F, Lee CY, Martin-Vega L (1986) Single machine scheduling with tight windows. Department of Industrial and System Engineering, University of Florida, Gainsville, Res. Rep 86-16

Biskup D, Feldmann M (2005) On scheduling around large restrictive common due windows. Eur J Oper Res 162:740-761

Bodin L, Golden B, Assad A, Ball M (1983) Routing and scheduling of vehicles and crews: the state of the art. Comput Oper Res 10:62-212

Burkard R, Cela E (1999) Linear assignment problems and extensions. In: Handbook of combinatorial optimization, Supplement vol A, Kluwer, Dordrecht, pp 75-149

Cheng T (1988) Optimal common due-date with limited completion times deviation. Comput Oper Res 15:91-96

Chu C, Gordon V, Proth JM (2002) Due date assignment and scheduling: SLK, TWK and other due date assignment models. Prod Plan Control 13:117-132

Gordon V, Proth JM, Chu C (2002) A survey of the state-of-the-art of common due date assignment and scheduling research. Eur J Oper Res 139:1-25

Hashimoto H, Yagiura M, Imahori S, Ibaraki T (2010) Recent progress of local search in handling the time window constraints of the vehicle routing problem. 4OR 8:221-238

Janiak A, Winczaszek M (2003) An optimal algorithm for a single processor scheduling problem with a common due window. In: Proceedings of 9th IEEE international conference on methods and models in automation and robotics, Miedzyzdroje, Poland, 25-28 August 2003, pp 1213-1216

Janiak A, Kovalyov M, Marek M (2007) Soft due window assignment and scheduling on parallel machines. IEEE Trans Syst Man Cybern Part A 37:614-620

Kacem I (2010) Fully polynomial time approximation scheme for the total weighted tardinessminimization with a common due date. Discret Appl Math 158:1035-1040

Kanet J, Sridharan V (2000) Scheduling with inserted idle time: problem taxonomy and literature review. Oper Res 48:99-110

Karakostas G, Kolliopoulos S, Wang J (2009) An fptas for the minimum total weightedtardiness problem with a fixed number of distinct due dates. In: Lecture notes in computer science, vol 5609. Springer, Berlin, pp 238-248. doi:10.1007/978-3-642-02882-3_24

Kellerer H, Strusevich V (2006) A fully polynomial approximation scheme for the singlemachine weighted total tardiness problem with a common due date. Theor Comput Sci 369:230-238

Koulamas C (1997) Maximizing the weighted number of on-time jobs in a single-machine scheduling with time windows. Math Comput Model 25:57-62

Kramer FJ, Lee CY (1994) Due window scheduling for parallel machines. Math Comput Model 20:69-89

Kritikos M, Ioannou G (2010) The balanced cargo vehicle routing problem with time windows. Int J Prod Econ 123:42-51

Lee CY (1991) Earliness-tardiness scheduling problems with constant size of due window. Department of Industrial and System Engineering, University of Florida, Gainsville, Res. Rep. 91-17

Li CL, Mosheiov G, Yovel U (2008) An efficient algorithm for minimizing earliness, tardiness, and due-date costs for equal-sized jobs. Comput Oper Res 35:3612-3619 
Li X, Tian P, Leung S (2010) Vehicle routing problems with time windows and stochastic travel and service times: models and algorithm. Int J Prod Econ 125:137-145

Liberatore F, Righini G, Salani M (2011) A column generation algorithm for the vehicle routing problem with soft time windows. 4OR 9:49-82

Mosheiov G (2001) A due-window determination in minmax scheduling problems. INFOR 39:107-123

Mosheiov G, Oron D (2004) Due-window assignment with unit processing-time jobs. Naval Res Logist 51:1005-1017

Mosheiov G, Sarig A (2010) Scheduling with a common due-window: polynomially solvable cases. Inf Sci 180:1492-1505

Schrage L (1981) Formulation and structure of more complex/realistic routing and scheduling problems. Networks 11:229-232

Solomon M, Desrosiers J (1988) Time window constrained routing and scheduling problems. Transp Sci 22:1-13

Torn A, Zilinskas A (1989) Global optimization. Lecture notes in computer science, vol 350. Springer, Heidelberg

Tuong NH, Soukhal A, Billaut JC (2010) A new dynamic programming formulation for scheduling independent tasks with common due date on parallel machines. Eur J Oper Res 202:646-653

Wang D, Fang S, Hodgson T (1998) A fuzzy due-date bargainer for the make-to-order manufacturing systems. IEEE Trans Syst Man Cybern Part C 28:492-497

Wang D, Fang S, Nuttle H (1999) Soft computing for multicustomer due-date bargaining. IEEE Trans Syst Man Cybern Part C 29:566-575

Wen C, Eksioglu S, Greenwood A, Zhang S (2010) Crane scheduling in a shipbuilding environment. Int J Prod Econ 124:40-50

Yen B, Wan G (1999) Single machine bicriteria scheduling: a survey. Int J Ind Eng Theory 10:222-231

Yeung W, Oguz C, Cheng T (2004) Two-stage flowshop earliness and tardiness machine scheduling involving a common due window. Int J Prod Econ 90:421-434 